\title{
True bilayer exciton condensate of one-dimensional electrons
}

\author{
A. Kantian and D. S. L. Abergel \\ Nordita, KTH Royal Institute of Technology and Stockholm University, \\ Roslagstullsbacken 23, SE-106 91 Stockholm, Sweden
}

(Dated: April 19, 2022)

\begin{abstract}
We theoretically predict that a true bilayer exciton condensate, characterised by off-diagonal long range order and global phase coherence, can be created in one-dimensional solid state electron systems. The mechanism by which this happens is to introduce a single particle hybridization of electron and hole populations, which locks the phase of the relevant mode and hence invalidates the MerminWagner theorem. Electron-hole interactions then amplify this tendency towards off-diagonal long range order, enhancing the condensate properties by more than an order of magnitude over the noninteracting limit. We show that the temperatures below which a substantial condensate fraction would form could reach hundreds of Kelvin, a benefit of the weak screening in one-dimensional systems.
\end{abstract}

Excitons are composite bosons formed from paired electrons and holes. They can be produced either by optical pumping of carriers between bands, or by bringing physically separate electrons and holes in close proximity. This latter type, called "bilayer excitons" occur because the mutual Coulomb interaction between the layers induces a many body instability, allowing the excitonic state to form. Under certain conditions, these composite bosons may condense into a Bose-Einstein condensate with off-diagonal long range order (ODLRO) and a global coherent phase [1]. Such a condensate has been observed for optically pumped excitons [2, and bilayer excitons in the quantum Hall regime [3. In zero magnetic field, exciton based generation of thermoelectricity has been proposed [4, and when condensed, bilayer excitons have been predicted to provide electrical transport across their bulk that is only limited by contacts and a linking resistor [5]. The predicted dissipationless current between layers is a direct result of the existence of the condensate and has been explored as the basis of valuable devices, such as ultra low power transistors [6].

However, a condensate of bilayer excitons in zero magnetic field has never been observed in an experiment on two-dimensional materials 7-11. Possible reasons include the critical temperature of the many body instability being simply too low, due to strong screening of the interlayer Coulomb interaction in two dimensions [12. Another reason might be the destruction of Fermi surface nesting by charged impurity disorder [13, 14]. Both of these factors could be mitigated by working with onedimensional (1D) bilayers, such as two parallel nanowires [15. Screening is known to be generally much weaker in 1D systems [16, 17, implying that the interlayer interaction would be more effective in 1D. As robustness against disorder derives from the magnitude of the order parameter [13, 14, this absence of screening would enhance the stability of the 1D exciton condensate (EC) in this respect as well.

The chief obstacle to any condensate of quantum particles in 1D is the Mermin-Wagner (MW) theorem, which prohibits spontaneous breaking of a continuous symmetry, and thus ODLRO, due to the enhancement of quantum fluctuations [18. In this work, we show that for 1D bilayer excitons, a very weak single particle tunneling between the two layers can lead to a true EC with ODLRO as the tunneling explicitly locks the phase of the relevant mode and thus the MW theorem no longer applies. Electron-hole attractions can then strongly feed into this small tendency towards ODLRO, resulting in large enhancements of all properties of the EC. This EC is a true many body condensate characterized by one large and one small excitation gap, both of which can be probed experimentally.

We employ highly accurate density matrix renormalization group (DMRG) numerics [19 to compute the ground and thermal state of the many body system. We show that the smaller gap sets the temperature scale on which crossover to the EC occurs. We also describe experimental probes of the EC by determining the nonlinear DC current-voltage characteristic of an interlayer transport measurement, and computing the density of states that would be probed in an STM experiment. Finally, we compute the ground states for systems with realistic length and energy scales and show that the EC can be realized at high temperatures after accounting for long range electron-electron interactions.

We consider a generic setup, two parallel quasi-1D electron systems ("wires", hence), shown in Fig. 1(a). Gates shift the electron bands such that the minimum of the conduction band for the upper wire is below the maximum of the valence band for the hole like lower wire. Weak interwire (IW) tunneling $t_{\perp}$ results in a joint chemical potential and, in the absence of interactions, the opening of a small single particle gap $\delta_{\mathrm{sp}}=2 t_{\perp}$ (Fig. 1(b)). To be compatible with DMRG, we consider a $1 \mathrm{D}$ space with $2 M$ lattice points ( $M$ points in each wire), corresponding either to real atoms in a 1D chain or to a districtized continuous $1 \mathrm{D}$ space. Introducing interactions, the Hamiltonian for this system is 


$$
\begin{array}{r}
\hat{H}=\hat{H}_{u}+\hat{H}_{l}+\hat{H}_{\mu}+\hat{H}_{\text {IWI }}+\hat{H}_{\text {IWT }} \text { with individual terms } \\
\hat{H}_{w}=-\sum_{x=1}^{M} t_{w}\left(\hat{c}_{x w}^{\dagger} \hat{c}_{x+1 w}+\text { h.c }\right) \\
+\sum_{x, y=1}^{M} U_{w}(|x-y|) \hat{n}_{x w} \hat{n}_{y w},
\end{array}
$$

and

$$
\hat{H}_{\mu}=\sum_{x=1}^{M} \frac{\mu_{\text {diff }}}{2}\left(\hat{n}_{x u}-\hat{n}_{x l}\right) .
$$

Here, $w \in\{u, l\}$ is the wire index, $\hat{c}_{x w}$ and $\hat{c}_{x w}^{\dagger}$ are electron field annihilators and creators at site $x$ in wire $w$, $\hat{n}_{x w}=\hat{c}_{x w}^{\dagger} \hat{c}_{x w}$, and $U_{u}=U_{l}$ is intrawire electron-electron interaction strength. The opposite band curvatures imply $t_{u}=-t_{l} \equiv t>0$, and the chemical potential difference $\mu_{\text {diff }}$ is used to tune the filling fraction of electrons inside each wire. The IW terms are

$$
\begin{aligned}
& \hat{H}_{\mathrm{IWI}}=\sum_{x, y=1}^{M} U_{u l}(|x-y|) \hat{n}_{x u} \hat{n}_{y l}, \\
& \hat{H}_{\mathrm{IWT}}=-t_{\perp} \sum_{x=1}^{M}\left(\hat{c}_{x u}^{\dagger} \hat{c}_{x l}+\text { h.c }\right) .
\end{aligned}
$$

where $U_{u l}$ is the IW interaction potential. To simplify the analysis and keep the required computational effort under control, we treat spinless electrons, as could be achieved, for example, by external magnetic fields (see Supplementary Materials).

It is the IW tunneling that enables exciton condensation in 1D. A particle-hole transformation for the hole wire shows $\hat{H}_{\text {IWT }}$ serving as bias field for electron-hole pairs. Without interactions, the ODLRO due to $\hat{H}_{\text {IWT }}$ is a trivial single particle effect brought on by the opening of the single particle gap $\delta_{\mathrm{sp}}$. In the following, we show that the IW repulsion between electrons $\hat{H}_{\text {IWI }}$ will feed strongly into this tiny nucleus of ODLRO and lead to a truly many body EC. This gives a massive enhancement of EC properties such as the temperature below which the system is close to the EC ground state, and the response to applying IW current and voltage. These properties are not affected by the explicit symmetry breaking nature of IW tunneling, which will attempt to fix the global condensate phase to a particular value. This phase locking effect was studied for bilayer systems, and most properties of the EC (including the technologically interesting ones) only rely on having a large condensate amplitude [5]. Treating the interplay of IW tunneling and electron interactions in 1D requires DMRG to fully capture the effects of nonperturbative $U_{u}, U_{l}$, and $U_{u l}$.

To illustrate the key features of the EC in $1 \mathrm{D}$, we first study a model system where electrons have no intrawire and purely local IW repulsion, so that $U_{u}=U_{l}=0$ and a)
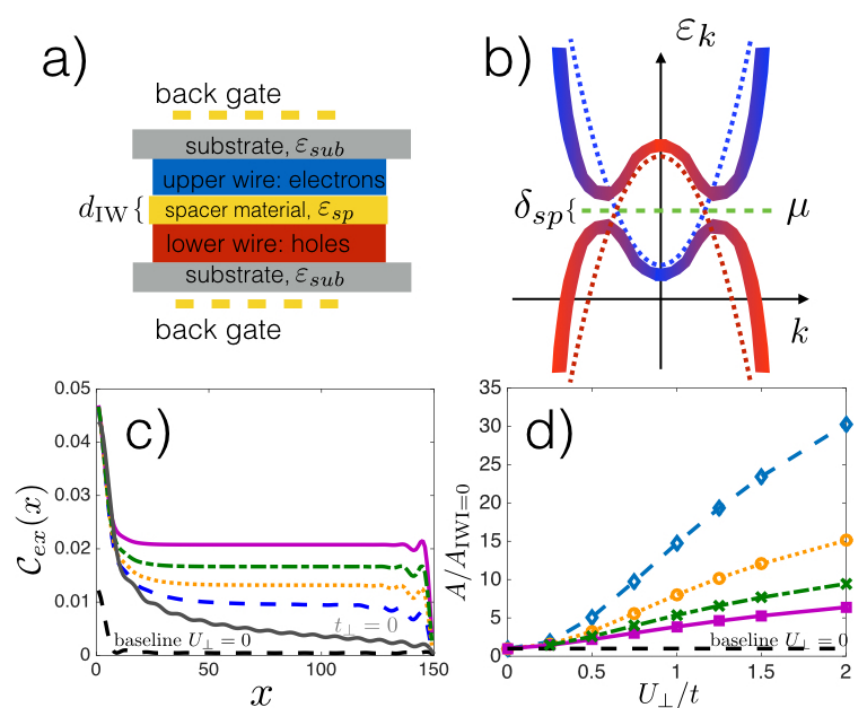

FIG. 1. (a) Sketch of the proposed system. (b) Band structure of noninteracting electrons in parallel nanowires with weak interwire tunneling $t_{\perp}$. The tunneling forces a gap $\delta_{\mathrm{sp}}=2 t_{\perp}$ (solid shaded lines) to open at the Fermi level. (c) Spatial dependence of the exciton-exciton correlator, showing the strong enhancement of excitonic off-diagonal long range order in the ideal model at zero temperature when $U_{\perp}=2 t$, for $t_{\perp}=0.001 t$ (blue dashed line), $t_{\perp}=0.0025 t$ (orange dotted) $t_{\perp}=0.005 t$ (green dash-dotted), $t_{\perp}=0.01 t$ (purple solid) $t_{\perp}=0$ (grey solid). Free electrons $\left(U_{\perp}=0\right)$ with $t_{\perp}=0.01$ (black dashed) shown for comparison. (d) Ratio of the order parameter $A$ of the exciton condensate with interactions $\left(U_{\perp} \neq 0\right)$ to noninteracting case (i.e. free fermions, $\left.U_{\perp}=0\right)$. In all cases, we see that sufficient $U_{\perp}$ can enhance the excitonic order by an order of magnitude or more. The line styles match (c). Results in (c) and (d) are for $M=300$.

$U_{u l}(|x-y|)=U_{\perp} \delta_{x, y}$. Once this is established, we show that with strong and long range intrawire interactions, a nontrivial and measurable EC still forms.

For the model system we calculate the ground states of $\hat{H}$ and their exciton correlations $\mathcal{C}_{\text {ex }}(x)=\left\langle\hat{c}_{0 u}^{\dagger} \hat{c}_{0 l} \hat{c}_{x l}^{\dagger} \hat{c}_{x u}\right\rangle$ for a grid of values of $t_{\perp}$ and $U_{\perp}$, fixing the filling fraction in the electron wire at 0.1 . In Fig. 1 1 (c) we plot $\mathcal{C}_{\text {ex }}(x)$ for $U_{\perp}=2 t$. The ODLRO is characterized by this exciton correlator approaching a finite value at long distances. When $t_{\perp}=0$ this cannot happen and $\mathcal{C}_{\text {ex }}(x)$ decays as $\propto x^{-K_{a}-1 / K_{s}}$, as predicted by bosonization and MW (see the Supplementary Material, and Ref. 20). In contrast, when $t_{\perp} \neq 0$ the exciton correlator remains finite at large $x$, indicating the presence of ODLRO and a stable EC. Decreasing $t_{\perp}$ by an order of magnitude only halves the strength of the ODLRO. Because DMRG uses a lattice with open boundaries, we see end effects where $\mathcal{C}_{\text {ex }}(x)$ oscillates on a scale inversely proportional to the small EC gap, $\delta$, described below. This is analogous to the penetration length of a superconductor. The noninteracting case, $U_{\perp}=0$, shown at $t_{\perp} \neq 0$ in Fig. 1(c) reveals the crucial importance of the IW interactions for enhancing 

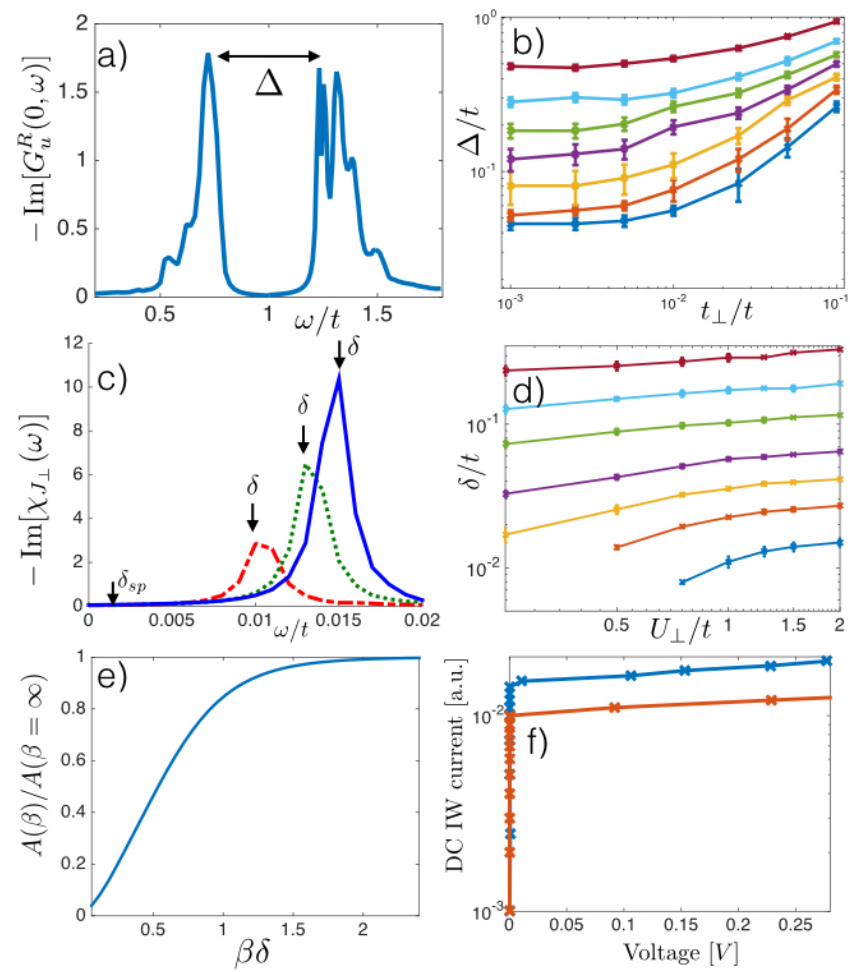

FIG. 2. (a) Spectral function of $G_{u}^{R}(x, \omega)$ for the model system with $t_{\perp}=0.001 t$ and $U_{\perp}=2 t$, exhibiting the large gap $\Delta$. (b) Scaling of $\Delta$ with $t_{\perp}$ for $U_{\perp}=0.25 t$ (dark blue), $U_{\perp}=0.5 t$ (bright red), $U_{\perp}=0.75 t$ (yellow), $U_{\perp}=t$ (violet) $U_{\perp}=1.25 t$ (green), $U_{\perp}=1.5 t$ (light blue), $U_{\perp}=2 t$ (dark red) for the model system. (c) Spectral function of $\chi_{J_{\perp}}(\omega)$ for the model system with $t_{\perp}=0.001 t$ and $\eta=0.001 t$, for $U_{\perp}=$ $2 t$ (blue line), $U_{\perp}=1.5 t$ (green dotted), and $U_{\perp}=t$ (red dash-dotted). Weight below $\delta$ is entirely due to finite $\eta$. (d) Scaling of $\delta$ with $U_{\perp}$, for $t_{\perp}=0.001 t$ (dark blue), $t_{\perp}=0.0025 t$ (bright red), $t_{\perp}=0.005 t$ (yellow), $t_{\perp}=0.01 t$ (violet) $t_{\perp}=$ $0.025 t$ (green), $t_{\perp}=0.05 t$ (light blue), $t_{\perp}=0.1 t$ (dark red) for the model system. (e) Order parameter $A$ as a fraction of its ground state value, against inverse temperature $\beta$ for the model system with $U_{\perp}=2 t, t_{\perp}=0.01 t$. Once $\beta>1 / \delta$, the system approaches ground state properties exponentially fast in $\beta$. (f) DC I-V characteristic of the model system with $t=1 \mathrm{eV}, U_{\perp}=2 t, t_{\perp}=0.01 t$ (blue) and $t_{\perp}=0.001 t$ (red), showing both dissipationless and dissipative regimes. All results are for $M=96$.

the magnitude of the ODLRO in the EC.

To quantify directly how electron interactions dominate the EC physics compared to the trivial gapped state of free electrons, Fig. 1(d) shows how the real space order parameter $A=\left\langle\hat{c}_{0 u}^{\dagger} \hat{c}_{0 l}\right\rangle$ of the EC is boosted over the corresponding value for $\hat{H}_{\mathrm{IWI}}=0$, which is set entirely by $t_{\perp}$. This order parameter also quantifies the ODLRO, since $\mathcal{C}_{\text {ex }}(x) \rightarrow A^{2}$ when $x \rightarrow \infty$.

Experimental observables capture how the IW interaction $U_{\perp}$ dominates the 1D EC physics. Fundamentally, the 1D EC is not characterised by one gap, but by two, which we label $\delta$ and $\Delta$. The large gap $\Delta$ could be mea- sured using scanning tunneling microscopy, which probes the retarded Green's function

$$
\begin{aligned}
G_{w}^{R}(x, \omega)=\left\langle\hat{c}_{x w}\left(\omega-\hat{H}+E_{G S}+i \eta\right)^{-1} \hat{c}_{x w}^{\dagger}\right\rangle \\
+\left\langle\hat{c}_{x w}^{\dagger}\left(\omega+\hat{H}-E_{G S}+i \eta\right)^{-1} \hat{c}_{x w}\right\rangle .
\end{aligned}
$$

An example is shown in Fig. 2(a). Weak coupling perturbative renormalization group (pRG) predicts $\Delta \propto U_{\perp}^{1 /\left(2-2 K_{a}\right)}$ (see the Supplementary Material and Ref. 20$)$, and is tied to the appearance of Coulomb drag [21 23. Numerically we find pRG to be of limited validity, with $\Delta\left(U_{\perp}\right)$ actually interpolating between (at least) two power laws in $U_{\perp}$, where the position of the crossover region depends on $t_{\perp}$ (see the Supplementary Material).

Crucially, DMRG reveals the dependence of $\Delta$ on $t_{\perp}$ (which pRG cannot), shown in Fig. 2(b). Two regimes of the $1 \mathrm{D}$ EC can be identified. At very small $t_{\perp} / t, \Delta$ is almost independent of $t_{\perp}$. Here the physics is almost completely dominated by electron-hole interactions and this is the cleanest form of a 1D many body EC. The other regime, when $t_{\perp} / t>0.005$, has a significant dependence of $\Delta$ on $t_{\perp}$ and a noticeable decrease of the order parameter ratio in Fig. 1(d), although that ratio still remains large if $U_{\perp} / t$ is large.

The large gap $\Delta$ is present even when $t_{\perp}=0$ and there is no EC. The small gap $\delta$ behaves differently. This gap can be obtained from the first peak in the imaginary part of the IW current susceptibility

$$
\chi_{J_{\perp}}(\omega)=\left\langle\hat{J}_{\perp}\left(\omega-\hat{H}+E_{G S}+i \eta\right)^{-1} \hat{J}_{\perp}\right\rangle,
$$

which is accessible via optical conductivity measurements and is shown in Fig. 2(c). Here, $\hat{J}_{\perp}=$ $\frac{i}{M} \sum_{x=1}^{M}\left(\hat{c}_{x u}^{\dagger} \hat{c}_{x l}-\right.$ h.c. $)$ is the discretized operator for IW current. We can also find $\delta$ by computing the first excited state above the ground state within the same quantum number sector [19] and this gives matching values. This gap only appears when $t_{\perp} \neq 0$ and is key for establishing the EC. The pRG predicts $\delta \propto t_{\perp}^{2 /\left(4-K_{a}+K_{s}^{-1}\right)}$ at weak $t_{\perp}$, and a locking of the phase of the symmetric mode (see the Supplementary Material and Ref. 20). However, pRG cannot characterize the order when both $t_{\perp}$ and $U_{\perp}$ flow to strong coupling, or when the system starts out at strong coupling. The limitations of pRG are illustrated again by our finding that $\delta\left(t_{\perp}\right)$ is not a pure power law, but consists of two such laws which cross over into each other (see the Supplementary Material). Thus, we have used DMRG to establish that the ordered phase of this system has excitonic ODLRO and the mutual enhancement of IW tunneling and interactions which pRG cannot deliver. The numerics further reveal that the EC order parameter $A \propto\left|\operatorname{Im}\left[\chi_{J_{\perp}}(\delta)\right]\right|^{\gamma}$ once $U_{\perp}$ becomes the dominant energy scale, where $\gamma$ is independent of $t_{\perp}$ (see the Supplementary Material). 


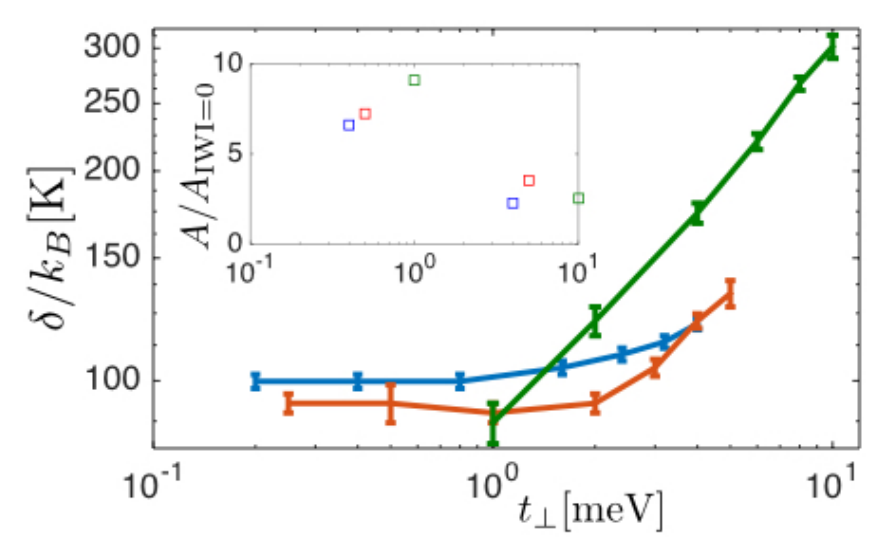

FIG. 3. Achievable gap $\delta / k_{B}$ in the strong screening case as a function of $t_{\perp}$ for $t=1 \mathrm{eV}$ (green line), $t=0.5 \mathrm{eV}$ (red line), and $t=0.4 \mathrm{eV}$ (blue line). Inset: range of achievable $A / A_{\mathrm{IWI}=0}$, indicated by showing high and low values as a function of $t_{\perp}$, with colors matching the main figure.

The gap $\delta$ sets the temperature below which the $1 \mathrm{D}$ excitons will be very close to the EC ground state, as shown by the condensate order parameter $A$ in Fig. 2(e). Computed quasiexactly using DMRG from the full thermal state $e^{-\beta \hat{H}}$ via the purification approach [19, $A(\beta)$ includes both the energy and entropy contributions to the free energy. We see that, even though it is very weak, the IW tunneling explicitly circumvents the standard argument of Landau and Lifshitz regarding the impossibility of an ordered EC phase at finite temperature, and $A$ in Fig. 2(e) exhibits crossover behaviour. This is analogous to the exact solution for the magnetization of a 1D Ising chain: at zero external magnetic field, no magnetized phase is possible at finite temperature, but any finite external field will give rise to a crossover behaviour of magnetization with temperature 24, exactly analogous to Fig. 2(e) for $A(\beta)$.

We calculate $\chi_{J_{\perp}}(\omega)$ in the real frequency domain (using the GMRES approach within DMRG 25]) on the isolated system. With no external bath to dissipate energy, this approach cannot obtain DC IW current in response to applying $\hat{J}_{\perp}$. Still, for an isolated system the existence of nondissipative DC interlayer supercurrent (which is the hallmark property for using the EC state as a transistor [6]) can be shown, as can the transition to a dissipative regime beyond some critical current. Both regimes are visible in Fig. 2(f), which shows $\chi_{J_{\perp}}(0)$ as a function of voltage $V=2 \pi \Gamma / e$, where $\Gamma$ is the rate of macroscopic tunneling from the original to the new ground state as $I \hat{J}_{\perp}$ is added to $\hat{H}$. We obtain $\Gamma$ from the decay of occupation from the original ground state through calculation of the imaginary time Green's function $\left\langle G S\left|e^{-\tau(\hat{H}+I \hat{J})}\right| G S\right\rangle \propto e^{-\tau \Gamma}$ using time dependent DMRG. The result agrees very well with the qualitative prediction of the singular relationship $I \propto-(\log V)^{-1}$.
Realistic experimental systems have long range Coulomb interactions, so now we demonstrate the robustness of the EC beyond the model system we have considered up to this point. We use a screened Coulomb potential (3D coordinates $\mathbf{x})$,

$$
U(|\mathbf{x}-\mathbf{x}|)=\frac{e^{-\left|\mathbf{x}-\mathbf{x}^{\prime}\right| / \Lambda}}{4 \pi \varepsilon_{\mathrm{eff}}\left|\mathbf{x}-\mathbf{x}^{\prime}\right|},
$$

where $\varepsilon_{\text {eff }}$ denotes the effective dielectric screening in between points $\mathbf{x}$ and $\mathbf{x}^{\prime}$. We note that for $1 \mathrm{D}$ electrons there is no intrinsic screening, a crucial advantage of implementing $1 \mathrm{D}$ excitons compared to previous proposals in 2D bilayers [12]. All screening in 1D derives from the environment [16, 17] and can thus be tuned. As shown in Fig. 1(a), the dielectric constant of the substrate $\varepsilon_{\text {sub }}$ could be different from that of the spacer $\varepsilon_{\mathrm{sp}}$ if different materials are chosen. For IW interactions $\varepsilon_{\mathrm{eff}}=\varepsilon_{\mathrm{sp}}$ and for intrawire interactions $\varepsilon_{\text {eff }}=\left(\varepsilon_{\mathrm{sub}}+\varepsilon_{\mathrm{sp}}\right) / 2$. The aim is to depress intrawire repulsion as much as possible through large $\varepsilon_{\text {sub }}$, while retaining strong IW repulsion through low $\varepsilon_{\mathrm{sp}}$. The particular form of the screening function in Eq. (1) is secondary: what matters for is to choose a screening that (i) limits the Coulomb interaction and (ii) reproduces the low energy properties of a realistic wire.

With this in mind, we consider a lattice model of electrons on two parallel chains, each with a lattice spacing $0.142 \mathrm{~nm}$ equal to the carbon-carbon bond of graphene and study two scenarios. (i) Moderate screening. Choosing $\varepsilon_{\text {sub }}=16 \varepsilon_{0}$ (where $\varepsilon_{0}$ is the vacuum permittivity), $\Lambda=0.48 \mathrm{~nm}$, and $t=0.25 \mathrm{eV}$, as explained in (Supplementary Material), a single such wire realizes system of strongly correlated spinless electrons at a magnetic field of $0.06 \mathrm{~T}$. Its low energy properties are characterized by a Tomonaga-Luttinger liquid parameter [18 $K=0.66$ (the model system had $K=1$ ), which is comparable to some experimentally available nanowires. We place two such wires $d_{\mathrm{IW}}=1 \mathrm{~nm}$ apart with $\varepsilon_{\mathrm{sp}}=\varepsilon_{0}$. Taking $t_{\perp}=0.25 \mathrm{meV}$, we use DMRG to compute the ground state and find that it exhibits ODLRO in $\mathcal{C}_{\text {ex }}$, that $\chi_{J_{\perp}}$ yields a substantial $\delta / k_{B}=88 \mathrm{~K}$, and that $A / A_{\mathrm{IWI}=0}=5.4$. For temperatures below $\delta$, this system will be very close to the EC ground state, having all the 1D EC properties established earlier. (ii) Strong screening. Choosing $\varepsilon_{\text {sub }}=200 \varepsilon_{0}, \Lambda=0.31 \mathrm{~nm}$, a wire is much closer to our initial model system than in the moderate screening case. When $d_{\mathrm{IW}}=0.75 \mathrm{~nm}$ and $\varepsilon_{\mathrm{sp}}=\varepsilon_{0}$, the IW interaction is larger than intrawire one. We consider systems at $t=0.4,0.5,1.0 \mathrm{eV}$, which can again be made spinless at perpendicular magnetic fields of $9.4 \mathrm{~T}$, $12 \mathrm{~T}$, and $27.9 \mathrm{~T}$ respectively (see Supplementary Materials). The IW-tunneling $t_{\perp}$ is varied between $5 \times 10^{-4} t$ and $0.01 t$. As shown in Fig. 3. this results in $\delta / k_{B}$ of at least $87 \mathrm{~K}$, reaching up to $301 \mathrm{~K}$. As for the model system earlier, we encounter both the regimes where $\delta$ depends weakly on $t_{\perp}$, crossing over into one where single particle 
physics becomes more noticeable. In the inset of Fig. 3 we indicate the range of the corresponding $A / A_{\mathrm{IWI}=0}$ by showing one high and one low value for each $t$. For small values of $t_{\perp}$ we see the many body physics being clearly dominant. We find that $U_{u}$ and $U_{l}$ should not be too different from $U_{u l}$ in overall magnitude. If intrawire interactions are much stronger than IW ones, all EC properties are depressed. In the opposite regime, where IW interactions dominate, the electrons phase separate at large $\mu_{\text {diff. }}$. As a result, we find different minimal filling fractions for the electrons in the upper wire (low filling aids pairing) For (i) this is 0.135 , in (ii) it is 0.27 at $t=0.4 \mathrm{eV}$, 0.208 at $t=0.5 \mathrm{eV}$, and 0.113 at $t=1 \mathrm{eV}$.

In conclusion, we have demonstrated that single particle tunneling between spinless electron and hole wires removes the restrictions of MW and allows for a true bilayer EC in one dimension characterized by ODLRO and a global phase.

Calculations were performed using the redeveloped DMRG module of ALPS [26] and the Matrix Product Toolkit [27. We thank Nordita for support. A.K. thanks Thierry Giamarchi for helpful discussions. D.S.L.A. thanks ERC project DM-321031 for financial support.

[1] S. A. Moskalenko and D. W. Snoke, Bose - Einstein Condensation of Excitons and Biexcitons (Cambridge University Press, 2000).

[2] D. Snoke, Science 298, 1368 (2002), ISSN 1095-9203, URL http://dx.doi.org/10.1126/science.1078082.

[3] J. P. Eisenstein and A. H. MacDonald, Nature 432, 691 (2004), ISSN 1476-4679, URL http://dx.doi.org/10. 1038/nature03081.

[4] K. Wu, L. Rademaker, and J. Zaanen, Phys. Rev. Appl. 2, 054013 (2014), ISSN 2331-7019, URL http://dx.doi. org/10.1103/PhysRevApplied.2.054013.

[5] J.-J. Su and A. H. MacDonald, Nat. Phys. 4, 799 (2008), ISSN 1745-2473, URL http://dx.doi.org/10. 1038/nphys 1055

[6] S. Banerjee, L. Register, E. Tutuc, D. Reddy, and A. MacDonald, IEEE Electron Device Lett. 30, 158 (2009), ISSN 1558-0563, URL http://dx.doi.org/10. 1109/LED . 2008.2009362.

[7] R. Pillarisetty, H. Noh, D. C. Tsui, E. P. De Poortere, E. Tutuc, and M. Shayegan, Physical Review Letters 89, 016805 (2002), ISSN 1079-7114, URL http://dx.doi. org/10.1103/PhysRevLett.89.016805

[8] J. A. Seamons, C. P. Morath, J. L. Reno, and M. P. Lilly, Physical Review Letters 102, 026804 (2009), ISSN 10797114, URL http://dx.doi.org/10.1103/PhysRevLett. 102.026804

[9] S. Kim, I. Jo, J. Nah, Z. Yao, S. K. Banerjee, and E. Tutuc, Physical Review B 83, 161401 (2011), ISSN 1550235X, URL http://dx.doi.org/10.1103/PhysRevB.83. 161401 .

[10] R. V. Gorbachev, A. K. Geim, M. I. Katsnelson, K. S. Novoselov, T. Tudorovskiy, I. V. Grigorieva, A. H. Mac-
Donald, S. V. Morozov, K. Watanabe, T. Taniguchi, et al., Nature Physics 8, 896 (2012), ISSN 1745-2481, URL http://dx.doi.org/10.1038/nphys2441

[11] A. Gamucci, D. Spirito, M. Carrega, B. Karmakar, A. Lombardo, M. Bruna, L. N. Pfeiffer, K. W. West, A. C. Ferrari, M. Polini, et al., Nat Comms 5, 5824 (2014), ISSN 2041-1723, URL http://dx.doi.org/10. $1038 /$ ncomms 6824

[12] I. Sodemann, D. A. Pesin, and A. H. MacDonald, Phys. Rev. B 85, 195136 (2012), URL http://dx.doi.org/10. 1103/PhysRevB.85.195136

[13] D. S. L. Abergel, R. Sensarma, and S. Das Sarma, Phys. Rev. B 86, 161412 (2012), ISSN 1550-235X, URL http: //dx.doi.org/10.1103/PhysRevB.86.161412

[14] D. S. L. Abergel, M. Rodriguez-Vega, E. Rossi, and S. Das Sarma, Phys. Rev. B 88, 235402 (2013), ISSN 1550-235X, URL http://dx.doi.org/10.1103/ PhysRevB.88.235402.

[15] D. S. L. Abergel, Appl. Phys. Lett. 106, 213103 (2015), ISSN 1077-3118, URL http://dx.doi.org/10.1063/1. 4921792

[16] H. J. Schulz, Phys. Rev. Lett. 71, 1864 (1993), ISSN 0031-9007, URL http://link.aps.org/doi/10.1103/ PhysRevLett.71.1864

[17] J. S. Meyer and K. A. Matveev, J. Phys.: Condens. Mat. 21, 023203 (2009), ISSN 0953-8984, URL http://stacks.iop.org/0953-8984/21/i=2/a=023203? key=crossref .04c619cb37173dbcf2f5842ea92251c5

[18] T. Giamarchi, Quantum Physics in One Dimension (Oxford University Press, 2003), 1st ed., ISBN 978-0-19852500-4.

[19] U. Schollwöck, Ann. Phys. 326, 96 (2011), ISSN 00034916, URL http://dx.doi.org/10.1016/j.aop. 2010.09.012

[20] Y. Werman and E. Berg, Phys. Rev. B 91, 245410 (2015), ISSN 1098-0121, URL http://link.aps.org/doi/10. 1103/PhysRevB.91.245410

[21] Y. V. Nazarov and D. V. Averin, Phys. Rev. Lett. 81, 653 (1998), ISSN 0031-9007, 9705158, URL http://link.aps.org/doi/10.1103/PhysRevLett.81. 653\{\%\}5Cnhttp://journals.aps.org/prl/abstract/ 10.1103/PhysRevLett.81.653\{\%\}5Cnhttp://journals . aps.org/prl/pdf/10.1103/PhysRevLett.81.653

[22] R. Klesse and A. Stern, Phys. Rev. B 62, 912 (2000).

[23] Y. Z. Chou, A. Levchenko, and M. S. Foster, Phys. Rev. Lett. 115, 1 (2015), ISSN 10797114, 1508.04433.

[24] L. E. Reichl, A modern course in statistical physics (Wiley, 2016), fourth rev ed., ISBN 3527413499.

[25] T. D. Kühner and S. R. White, Phys. Rev. B 60, 335 (1999), ISSN 0163-1829, URL http://link.aps.org/ doi/10.1103/PhysRevB.60.335

[26] M. Dolfi, B. Bauer, S. Keller, A. Kosenkov, T. Ewart, A. Kantian, T. Giamarchi, and M. Troyer, Comput. Phys. Commun. 185, 3430 (2014), ISSN 00104655, URL http://www.sciencedirect.com/science/article/ pii/S0010465514003002

[27] URL https://people.smp.uq.edu.au/IanMcCulloch/ mptoolkit/ 


\section{SUPPLEMENTARY MATERIAL}

\section{Perturbative RG of the bosonized model}

Though treating $\hat{H}_{\text {IWI }}$ and $\hat{H}_{\text {IWT }}$ perturbatively will not address physically relevant systems, together with the associated bosonization framework [18] it does have some use for interpreting the numerical results at strong coupling. After a particle-hole transformation $\hat{c}_{x l} \rightarrow(-1)^{x} \hat{c}_{x l}^{\dagger}$ on $\hat{H}_{l}$, we bosonize $\hat{H}_{u}+\hat{H}_{l}$, by retaining only the long wavelength excitations around the Fermi points $\pm k_{F}$ in both wires, approximating $\hat{c}_{x w} \propto U_{R} e^{i\left(k_{F} x-\hat{\phi}_{w}(x)+\hat{\theta}_{w}(x)\right)}+U_{L} e^{-i\left(k_{F} x-\hat{\phi}_{w}(x)-\hat{\theta}_{w}(x)\right)}$, where $\hat{\phi}_{w}(x), \partial_{x} \hat{\theta}_{w}(x)$ are conjugate field operators, $\left[\hat{\phi}_{w}(x), \partial_{x} \hat{\theta}_{w^{\prime}}\left(x^{\prime}\right)\right]=i \pi \delta_{w w^{\prime}} \delta\left(x-x^{\prime}\right)$ associated with long wavelength density and phase fluctuations in wire $w$ respectively, and $U_{R}$ and $U_{L}$ are the Klein factors that preserve anticommutation relations. Thus, $\hat{H}_{w}$ becomes quadratic in $\partial_{x} \hat{\phi}_{w}(x), \partial_{x} \hat{\theta}_{w}(x)$ and its long wavelength properties are parametrised by just two numbers, the Tomonaga-Luttinger liquid (TLL) parameters $v_{w}$ and $K_{w}$. The TLL parameters $K_{w}$ encode the strength and range of $U_{w}$ respectively and if $U_{w}=0$, then $K_{w}=1$. The stronger and more long ranged $U_{w}$ is, the further below 1 the value of $K_{w}$ will drop.

Since we assume $\left|t_{u}\right|=\left|t_{l}\right|, U_{u}=U_{l}$, and $k_{F}$ being the same for both wires, we have $K_{u}=K_{l}=K$ in the following. Now adding $\hat{H}_{\text {IWI }}$ as perturbation to $\hat{H}_{u}+\hat{H}_{l}$, its bosonized form in momentum space decomposes into a forward scattering part, with terms proportional to $U_{u l}^{F}=U_{u l}(q=0)$ and a backscattering contribution proportional to $U_{u l}^{B}=U_{u l}\left(q=2 k_{F}\right)$. The forward scattering term can be incorporated into $\hat{H}_{u}+\hat{H}_{l}$ exactly, at the price of a canonical transformation to the symmetric and antisymmetric modes of the two wires $\hat{\phi}_{s, a}=\left(\hat{\phi}_{u} \pm \hat{\phi}_{l}\right) / \sqrt{2}, \hat{\theta}_{s, a}=\left(\hat{\theta}_{u} \pm \hat{\theta}_{l}\right) / \sqrt{2}$. This results in $\hat{H}_{u}+\hat{H}_{l} \rightarrow \hat{H}_{s}+\hat{H}_{a}$, where $\hat{H}_{s}$ and $\hat{H}_{a}$ are TLL Hamiltonians, and in perturbation theory their TLL parameters are $K_{s, a}=\left((K)^{-2} \mp U_{u l}^{F} m_{u} /\left(2 \hbar k_{F} K\right)\right)^{-1 / 2}$. However, the backscattering part can at best be treated using the perturbative renormalization group ( $\mathrm{pRG}$ ), and the same holds for the bosonized version of $\hat{H}_{\mathrm{IWT}}$. On its own, the IW backscattering (when relevant) is the source of Coulomb drag [21]23].

As in Ref. [20], treating both perturbations jointly in second-order momentum space pRG gives

$$
\frac{d U_{u l}^{B}}{d s}=2\left(1-K_{a}\right) U_{u l}^{B}, \quad \frac{d t_{\perp}}{d s}=\frac{\left(4-K_{a}-K_{s}^{-1}\right) t_{\perp}}{2} .
$$

Thus, both $\hat{H}_{\text {IWI }}$ and $\hat{H}_{\text {IWT }}$ are relevant perturbations for a very wide range of parameters (for example, $\hat{H}_{\text {IWI }}$ is so for any repulsive $U_{u l}$ ), and their associated couplings both flow to nonperturbative values, outside the range of any pRG. The validity of Eq. (2) is constrained further because the $U_{u l}^{B}$ may flow to its fixed point be-
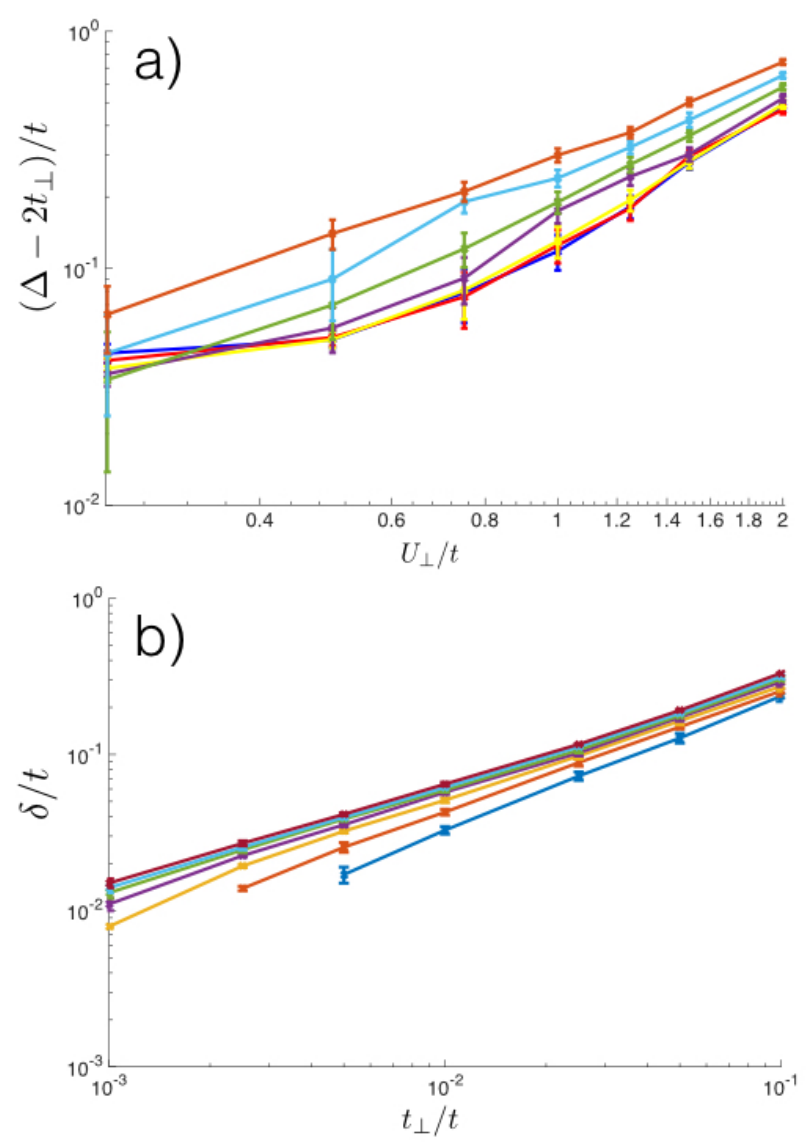

FIG. 4. (a) Interaction-dependent part of the gap $\Delta, \Delta-$ $2 t_{\perp}$, plotted against $U_{\perp}$ for the ideal model system, at $t_{\perp}=$ $0.001 t$ (dark blue), $t_{\perp}=0.0025 t$ (light red), $t_{\perp}=0.005 t$ (yellow),$t_{\perp}=0.01 t$ (violet), $t_{\perp}=0.025 t$ (green), $t_{\perp}=0.05 t$ (light blue), $t_{\perp}=0.1 t$ (dark red). (b) Gap $\delta$ against $t_{\perp}$ for the ideal model system, at $U_{\perp}=0.25 t$ (dark blue), $U_{\perp}=0.5 t$ (light red), $U_{\perp}=0.75 t$ (yellow), $U_{\perp}=t$ (violet), $U_{\perp}=1.25 t$ (green), $U_{\perp}=1.5 t$ (light blue), $U_{\perp}=2 t$ (dark red).

fore $t_{\perp}$, locking $\hat{\phi}_{a}$ to a fixed value and making the $\mathrm{pRG}$ equation for $t_{\perp}$ obsolete. If, against these objections, a straight extrapolation of Eq. (2) is performed, it would predict one gap $\Delta$ for fluctuations of $\hat{\phi}_{a}$, with scaling $\Delta \sim\left(U_{u l}^{B}\right)^{1 / 2\left(1-K_{a}\right)}$, and another, $\delta$, for fluctuations of $\hat{\theta}_{s}$, with scaling $\delta \sim t_{\perp}^{2 /\left(4-K_{a}+K_{s}^{-1}\right)}$, the most relevant bosonized operators inside $\hat{H}_{\text {IWI }}$ and $\hat{H}_{\text {IWT }}$ being compatible.

\section{Comparison to DMRG - beyond perturbative RG}

With DMRG numerics we can address the regimes in which pRG is not valid. The main text focuses on the most relevant effects beyond pRG, such as the establishment of excitonic ODLRO, the mutual reinforcement of 


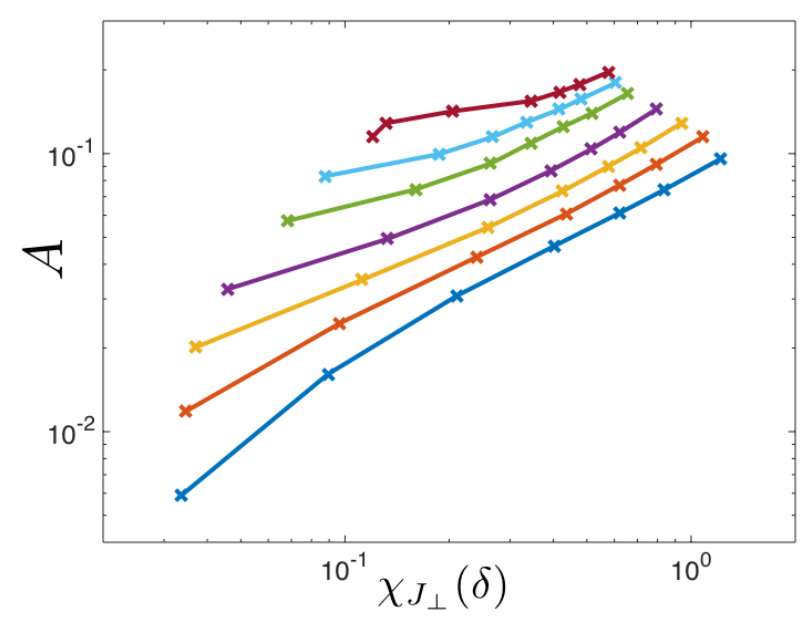

FIG. 5. Order parameter $A$ as a function of the peak of the IW conductivity $\chi_{J_{\perp}}$ at $\omega=\delta$, for $t_{\perp}=0.001 t$ (dark blue), $t_{\perp}=0.0025 t$ (light red), $t_{\perp}=0.005 t$ (yellow), $t_{\perp}=0.01 t$ (violet), $t_{\perp}=0.025 t$ (green), $t_{\perp}=0.05 t$ (light blue), $t_{\perp}=$ $0.1 t$ (dark red). All data taken at $\eta=0.01 t$.

IW interaction and IW tunneling leading to enhancements of both $\delta$ and $A$, as well as computing $\delta$ quantitatively for quasirealistic systems,

We compare DMRG to the predictions that pRG can be used for, the scaling of $\Delta$ with $U_{u l}^{B}$, and of $\delta$ with $t_{\perp}$ for our ideal model system with $U_{u}=U_{l}=0, U_{u l}^{B}=U_{\perp}$. As can be seen in Fig. (4(a), $\Delta$ is governed by at least two different power laws (as opposed to the single power law predicted by $\mathrm{pRG}$ ) and the position of the crossover between the two power laws strongly depends on $t_{\perp}$. Note that we have subtracted the trivial contribution to the gap $\Delta$ which comes from the band hybridization and is equal to $2 t_{\perp}$.

For the gap $\delta$ we find qualitatively similar results, as shown in Fig. 4 (b), but we only enter the crossover zone for the lowest values of $U_{\perp}$ and $t_{\perp}$ within our parameter grid.

\section{Linking $A$ to observables}

We show in the main text that the peak of the IW conductivity $\chi_{J_{\perp}}(\omega)$ at $\omega=\delta$ is a proxy for the order parameter $A$ of the 1D EC, and is the figure of merit for proposed applications of bilayer ECs as low power electrical switches. We show in Fig. 5, that as $\chi_{J_{\perp}}(\omega)$ increases with $U_{\perp}$ for any given $t_{\perp}$ (the lines in Fig. 5 correspond to different values of $t_{\perp}$ ), the dependency of $A$ on $\chi_{J_{\perp}}(\delta)$ seems to enter a power law scaling with an exponent $\gamma$. Within the resolution of our grid of $\omega$ values, the value of $\gamma$ for each $t_{\perp}$ appears to be either the same, or so close to each other that we cannot resolve the difference.

\section{Obtaining a spin-polarized system}

For the quasi-realistic systems with long range interactions (Eq. (1) of the main text), the magnetic field needed to prepare the system in the fully spin-polarized state that we study can be straightforwardly computed using DMRG. We calculate the energy of the ground state $E_{\mathrm{GS}}\left(N_{\uparrow}=N_{\downarrow}\right)$ in the spin-balanced system, as well as for the spin-polarized system, $E_{\mathrm{GS}}\left(2 N_{\downarrow}\right)$. The long range interactions are exactly the same in each case, but in our discretized spinful wire we assume an onsite repulsion $U / t=10$. The magnetic Zeeman energy of an electron in the spinful system with an external magnertic field $B$ perpendicular to the wires is given by $E_{\mathrm{mag}}(\sigma)=g \mu_{B} B \sigma$, with $\sigma= \pm 1 / 2$, the Lande $g$-factor $g=2$ and the Bohr magneton $\mu_{B}=5.788 \times 10^{-5} \mathrm{eV} / \mathrm{T}$. Thus, at

$$
B_{\mathrm{pol}}=\frac{E_{\mathrm{GS}}\left(2 N_{\downarrow}\right)-E_{\mathrm{GS}}\left(N_{\uparrow}, N_{\downarrow}\right)}{g \mu_{B} N_{\downarrow}}
$$

the magnetic field is strong enough to polarize both wires.

Thus, in the non-polarized case at $B=0$, one would start with the spin-degenerate electron-like bands of the upper wire overlapping with the hole-like bands of the lower wire, but at the same time the Fermi level would be below the band minimum of the upper wire. Generically, it is possible to achieve this by employing back gates. Then, as $B$ becomes non-zero, the spin-down bands of both wires will be shifted down in energy until the Fermi level lies in the hybridization gap of the spin-down bands. This is the situation depicted in Fig. 1b of the main text, and as long as $B>B_{\text {pol }}$ holds, this system is fully spinpolarized. 